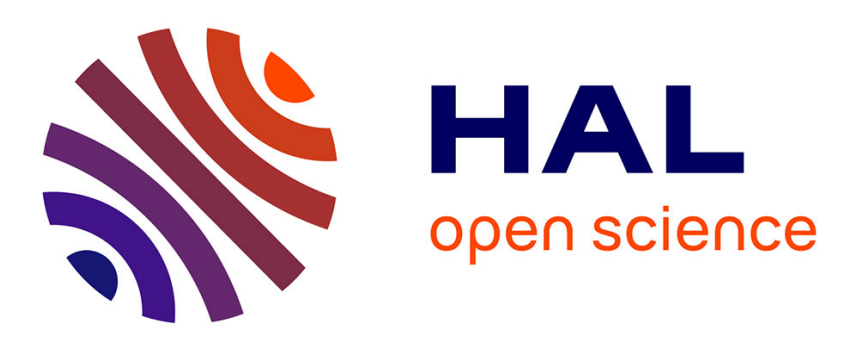

\title{
On the linear combination of the Gaussian and student's $t$ random field and the integral geometry of its excursion sets
}

Ola Suleiman Ahmad, Jean-Charles Pinoli

\section{- To cite this version:}

Ola Suleiman Ahmad, Jean-Charles Pinoli. On the linear combination of the Gaussian and student's t random field and the integral geometry of its excursion sets. Statistics and Probability Letters, 2013, 83, pp.559-567. 10.1016/j.spl.2012.10.022 . hal-00819094

\section{HAL Id: hal-00819094 https://hal.science/hal-00819094}

Submitted on 3 May 2013

HAL is a multi-disciplinary open access archive for the deposit and dissemination of scientific research documents, whether they are published or not. The documents may come from teaching and research institutions in France or abroad, or from public or private research centers.
L'archive ouverte pluridisciplinaire HAL, est destinée au dépôt et à la diffusion de documents scientifiques de niveau recherche, publiés ou non, émanant des établissements d'enseignement et de recherche français ou étrangers, des laboratoires publics ou privés. 


\title{
On the linear combination of the Gaussian and student's $t$ random field and the integral geometry of its excursion sets
}

\author{
Ola Ahmad*, Jean-Charles Pinoli* \\ LGF, UMR CNRS 5146
}

Ecole Nationale Supérieure des Mines de Saint-Etienne

158 cours Fauriel, F-42023 Saint-Etienne cedex 2, France

\begin{abstract}
In this paper, a random field, denoted by $G T_{\beta}^{\nu}$, is defined from the linear combination of two independent random fields, one is a Gaussian random field and the second is a student's $t$ random field with $\nu$ degrees of freedom scaled by $\beta$. The goal is to give the analytical expressions of the expected Euler-Poincaré characteristic of the $G T_{\beta}^{\nu}$ excursion sets on a compact subset $S$ of $\mathbb{R}^{2}$. The motivation comes from the need to model the topography of 3D rough surfaces represented by a 3D map of correlated and randomly distributed heights with respect to a $G T_{\beta}^{\nu}$ random field. The analytical and empirical Euler-Poincaré characteristic are compared in order to test the $G T_{\beta}^{\nu}$ model on the real surface.
\end{abstract}

Keywords: Gaussian random field, Student's $t$ random field, Excursion sets, Minkowski functionals, Euler-Poincaré characteristic

\footnotetext{
*Corresponding author.

Email address: ahmad@emse.fr (Ola Ahmad)
} 


\section{Introduction}

The motivation of studying the linear combination between the Gaussian and the student's $t$ random fields, $G T_{\beta}^{\nu}$, comes from the need to model some examples of 3D rough engineering surfaces used in biomedical and material science applications.

Studying the spatial evolution of a surface or the deformation of its peaks requires combination between different types of random fields. This combination might increase the flexibility of the model, since it defines further statistical parameters that enable describing the shape of the height's distribution, (i.e. the skewness, the kurtosis,...etc.), which could interpret the functionality of such surfaces during certain phenomenons, when they cannot be involved by only the Gaussian random fields.

Gaussian and several non-Gaussian random fields, including, without limitation, $\chi^{2}, F$, student's $t$ and Hotelling's $t^{2}$ random fields, have been studied by (Adler, 1981; Adler and Taylor, 2007; Alder and Taylor, 2003; Cao and Worsley, 2001; Worsley, 1995) in order to detect the p-value of the local maxima of a random field inside a searching region that correspond to a certain activation in the brain or for searching certain anomalies in medical imaging applications. For this aim, the integral geometric characteristics of the excursion sets of such random fields have been investigated in (Adler and Taylor, 2011; Adler, 1981; Cao, 1997; Worsley, 1995). The excursion sets are defined as the upper sets that result from thresholding the random field at a given level $h$. For example, the excursion set at a height level $h$ of a 3D surface defined on a two-dimensional space will result from hitting the surface heights with a plane at the level $h$. Thus, all the heights that exceed the threshold $h$ will belong to this excursion set. The integral geometry of the excursion sets is of great interest, since it defines their Euler, or Euler-Poincaré characteristic, which counts, on a com- 
pact subset of two dimensions, the number of connected components of the excursion set, minus the number of holes. This characteristic function enables detecting the peaks and the valleys elevations on 3D rough surfaces, and so, it describes the surface roughness.

In this paper, we are interested in studying the linear combination of a stationary Gaussian random field, denoted by $G$, and a non-Gaussian random field, namely student's $t$ random field with $\nu$ degrees of freedom, denoted by $T^{\nu}$, i.e., the sum $G(x)+\beta T^{\nu}(x)$, where $x$ stands for the spatial location that belongs to a subset $S$ of $\mathbb{R}^{2}$. This random field will be denoted by $G T_{\beta}^{\nu}$. The goal is to introduce the stationary $G T_{\beta}^{\nu}$ random field, and to calculate analytically the expected Euler-Poincaré characteristic of its excursion set on a rectangle of $\mathbb{R}^{2}$.

The paper is organized as follows. Firstly, the $G T_{\beta}^{\nu}$ random field is defined, (section 2), with its distribution function. Secondly, the expected Euler-Poincaré characteristic of the $G T_{\beta}^{\nu}$ excursion sets is expressed analytically on $\mathbb{R}^{2}$ (section 3 ) . An application is reported on a $3 \mathrm{D}$ rough surface, (section 4 ), in order to test the $G T_{\beta}^{\nu}$ random field on the height's map, and to describe the surface roughness.

\section{2. $G T_{\beta}^{\nu}$ random fields}

\subsection{Notation}

Let $Y(x)$ be a real-valued random field, $\{Y=Y(x), x \in S\}$, represented at a point $x$ in a compact subset $S$ of the Euclidean space $\mathbb{R}^{d}$, with mean $\mu_{Y}$ and variance $\sigma_{Y}^{2}$. The $N \times N$ covariance matrix of any arbitrary finite collection $\left\{Y_{i}=Y\left(x_{i}\right), i \leq 1 \leq N, x_{i} \in S\right\}$, will be denoted by $C_{Y}$, where $C_{Y}(i, j)=\mathbb{E}\left[\left(Y_{i}-\right.\right.$ $\left.\left.\mu_{Y_{i}}\right)\left(Y_{j}-\mu_{Y_{j}}\right)\right],(i, j=1, \ldots, N)$. The probability density function of $Y$, at any point $x$, 
will be denoted by $p_{Y}$ and the cumulative distribution function will be denoted by $P_{Y}$.

\subsection{Univariate distribution}

Definition 1 ( $G T_{\beta}^{\nu}$ random variable). Let $G$ be a random variable of standard normal distribution and $T^{\nu}$ be a zero mean student's $t$ random variable with $\nu$ degrees of freedom independent of $G$. A random variable $Y$ is said to have a $G T_{\beta}^{\nu}$ distribution if it is defined from the sum $Y=G+\beta T^{\nu}, \beta \in \mathbb{R} \backslash\{0\}$. It will be denoted by $\left(Y \sim G T_{\beta}^{\nu}\right)$.

The probability density function of $Y,\left(p_{Y}(h)\right)$, is then derived from the convolution between the probability density functions of $G$ and $T^{\nu}$ as follows:

$$
p_{Y}(h)=\left(p_{G} * p_{T_{\beta}^{\nu}}\right)(h)=\frac{\Gamma\left(\frac{\nu+1}{2}\right)}{\beta \pi \Gamma\left(\frac{\nu}{2}\right) \sqrt{2 \nu}} \int_{-\infty}^{\infty}\left(1+\frac{(h-u)^{2}}{\beta^{2} \nu}\right)^{-\frac{\nu+1}{2}} e^{-\frac{u^{2}}{2}} d u,
$$

where

$$
p_{G}(h)=\frac{1}{\sqrt{2 \pi}} e^{-h^{2} / 2}
$$

is the normal probability density function, and

$$
p_{T^{\nu}}(h)=\frac{\Gamma\left(\frac{\nu+1}{2}\right)}{\beta \Gamma\left(\frac{\nu}{2}\right) \sqrt{\pi \nu}}\left(1+\frac{h^{2}}{\nu \beta^{2}}\right)^{-(\nu+1) / 2}
$$

is the probability density function of the student's $t$ distribution with $\nu$ degrees of freedom and scaled by $\beta$.

The cumulative distribution function $P_{Y}(h)=\mathbb{P}[Y \leq h]$ is given by:

$$
\begin{aligned}
\mathbb{P}[Y \leq h] & =\int_{-\infty}^{h} p_{Y}(u) d u=\int_{-\infty}^{h}\left(p_{G} * p_{T_{\beta}^{\nu}}\right)(u) d u \\
& =\frac{\Gamma\left(\frac{\nu+1}{2}\right)}{2 \pi \beta \Gamma\left(\frac{\nu}{2}\right) \sqrt{\nu / 2}} \int_{-\infty}^{h} \int_{-\infty}^{\infty}\left(1+\frac{(u-v)^{2}}{\beta^{2} \nu}\right)^{-\frac{\nu+1}{2}} e^{-\frac{v^{2}}{2}} d u d v \\
& =\frac{1}{\sqrt{2 \pi}} \int_{-\infty}^{h} \int_{-\infty}^{\infty} v e^{-\frac{v^{2}}{2}} P_{T^{\nu}}\left(\frac{u-v}{\beta}\right) d u d v
\end{aligned}
$$


where $P_{T^{\nu}}$ is the cumulative distribution function of the student's $t$ distribution with $\nu$ degrees of freedom and scaled by $\beta$.

It could be noticed that $P_{Y}(h)$ will converge to the Gaussian cumulative distribution $P_{G}(h)$ when $\beta$ tends toward zero :

$$
\begin{aligned}
\lim _{\beta \rightarrow 0} P_{Y}(h) & =\lim _{\beta \rightarrow 0} \frac{1}{\sqrt{2 \pi}} \int_{-\infty}^{h} \int_{-\infty}^{\infty} v e^{-\frac{v^{2}}{2}} P_{T^{\nu}}\left(\frac{u-v}{\beta}\right) d u d v \\
& =\frac{1}{\sqrt{2 \pi}} \int_{-\infty}^{h} \int_{0}^{\infty}(u-s) e^{-\frac{(u-s)^{2}}{2}} d u d s \\
& =\frac{1}{\sqrt{2 \pi}} \int_{-\infty}^{h} e^{-\frac{u^{2}}{2}} d u=P_{G}(h)
\end{aligned}
$$

where

$$
\lim _{\beta \rightarrow 0}\left[P_{T^{\nu}}\left(\frac{s}{\beta}\right)\right]= \begin{cases}1 & s>0 \\ 0 & s \leq 0\end{cases}
$$

In the general case, $Y$ might be centered around a mean value $\mu_{Y}$ and scaled by $\sigma_{Y}$ such that $Y=\mu_{Y}+\sigma_{Y}\left(G+\beta T_{s}^{\nu}\right)$. This yields applying the transformation $h \rightarrow \frac{h-\mu_{Y}}{\sigma_{Y}}$ in order to obtain the $G T_{\beta}^{\nu}$ probability density function as follows:

$$
p_{Y}(h)=\frac{1}{\sigma_{Y}}\left(p_{G} * p_{T_{\beta}^{\nu}}\right)\left(\frac{h-\mu_{Y}}{\sigma_{Y}}\right)
$$

\subsection{Multivariate distribution}

Assume $\mathbf{Y}$ is a vector of $N$ random variables $\mathbf{Y}=\left(Y_{1}, \ldots, Y_{N}\right)^{t},(N>1)$, then $\mathrm{Y}$ is said to have an $N$-dimensional multivariate $G T_{\beta}^{\nu}$ distribution, denoted by $p_{\mathbf{Y}}\left(\mathbf{h} ; C_{Y}\right), \mathbf{h} \in \mathbb{R}^{N}$, with mean vector $\left(\mu_{\mathbf{Y}}=\left(\mu_{Y_{1}}, \ldots, \mu_{Y_{N}}\right)^{t}\right)$ and $(N \times N)$ covariance matrix $C_{Y}$, if each component, $Y_{i}$, defines a $\left(G T_{\beta}^{\nu}\right)_{i}$ random variable, such that:

$$
Y_{i}=\mu_{Y_{i}}+\left(G_{i}+\beta_{i} T_{i}^{\nu}\right), \quad i=1, \ldots, N
$$


then the covariance matrix $C_{Y}$ is:

$$
C_{Y}=C_{G}+\frac{\nu}{\nu-2} \beta C \beta^{t}, \nu>2
$$

where $C_{G}, C$ are the $(N \times N)$ covariance matrix of the Gaussian and the student's $t$ multivariate distributions, respectively, and $\beta=\left(\beta_{1}, \ldots, \beta_{N}\right)^{t}$.

\section{4. $G T_{\beta}^{\nu}$ random field}

On a subset $S$ of $\mathbb{R}^{d}$, if any arbitrary collection of $N$ random variables, $\left(Y\left(x_{1}\right), \ldots, Y\left(x_{N}\right)\right)$ at any choice $\left(x_{1}, \ldots, x_{N}\right) \in S$, has a $G T_{\beta}^{\nu}$ multivariate distribution, then for any $x \in S, Y(x)$ will define a $G T_{\beta}^{\nu}$ random field with $\nu$ degrees of freedom, which yields to the following definition :

Definition 2 ( $G T_{\beta}^{\nu}$ random field). Let $G$ be a stationary isotropic Gaussian random field on a compact subset $S \subset \mathbb{R}^{d}$ with zero mean, $\mu_{G}=0$, and unit variance $\sigma_{G}^{2}=$ 1. Let $T^{\nu}$ be a homogeneous student's $t$ random field with $\nu$ degrees of freedom, independent of $G$. Then, the sum given by:

$$
Y(x)=G(x)+\beta T^{\nu}(x), \quad \beta \in \mathbb{R} \backslash\{0\}
$$

defines a stationary $G T_{\beta}^{\nu}$ real-valued random field with $\nu$ degrees of freedom.

\section{The expected Euler-Poincaré characteristic of the $G T_{\beta}^{\nu}$ excursion sets}

The expected Euler-Poincaré characteristic of both Gaussian and student's $t$ excursion sets has been expressed by its explicit analytical formula in (Adler, 1981; Adler et al., 2010; Cao and Worsley, 2001; Worsley, 1994) on $\mathbb{R}^{d}$, for $d=1,2,3$. In this paper, we will focus on estimating the expected Euler-Poincaré characteristic of the $G T_{\beta}^{\nu}$ excursion sets in a compact subset $S$ of $\mathbb{R}^{2}$. 


\subsection{Preliminaries}

1. Let $Y(x), x \in \mathbb{R}^{2}$, be a zero mean real-valued $G T_{\beta}^{\nu}$ random field given by the sum $G(x)+\beta T^{\nu}(x)$ and defined on a compact subset $S \subset \mathbb{R}^{2} . G$ is supposed to be a stationary isotropic Gaussian random field of zero mean, $\mu_{G}=0$, unit variance, $\sigma_{G}^{2}=1$, and with a $(2 \times 2)$ covariance matrix $C_{G}$. $T^{\nu}$ is a homogeneous student's $t$ random field with $\nu>2$. In the following, $T^{\nu}$ will be represented in terms of its independent Gaussian components, at any point fixed point $x \in S$, (Worsley, 1994), such that:

$$
T^{\nu}(x)=\frac{\sqrt{\nu} X_{0}(x)}{\left[\sum_{k=1}^{\nu} X_{k}^{2}(x)\right]^{1 / 2}}
$$

where $X_{k},(k=0, \ldots, \nu)$, are $\nu+1$ independent, homogeneous, and identically distributed Gaussian random fields, on $\mathbb{R}^{2}$, of zero means, unit variance, and with a $(2 \times 2)$ covariance matrix $C$ such that $X_{k} \sim \operatorname{Normal}_{2}(0, C)$.

Let $E_{h}(Y, S)$ be the excursion set, Adler (1981), of $Y$ inside $S$, above a given threshold $h$, which is defined as follows:

$$
E_{h}(Y, S)=\{x \in S: Y(x) \geq h\}
$$

2. Let $\Lambda_{G}$ be the $(2 \times 2)$ variance-covariance matrix of $G$ (i.e., the covariance matrix between the first order partial derivatives $\nabla G$, or in other words, the second order partial derivative of the covariance function $C_{G}$ at zero, (Alder and Taylor, 2003)) whose elements are expressed as:

$$
\lambda_{G_{i j}}=\mathbb{E}\left[\frac{\partial G}{\partial x_{i}} \frac{\partial G}{\partial x_{j}}\right]=-\left.\frac{\partial^{2} C_{G}(x)}{\partial x_{i} \partial x_{j}}\right|_{x=0}
$$

and $\Lambda$ be the $(2 \times 2)$ variance-covariance matrix of any $X_{k},(k=0, \ldots, \nu)$ such that:

$$
\lambda_{i j}=\mathbb{E}\left[\frac{\partial X_{k}}{\partial x_{i}} \frac{\partial X_{k}}{\partial x_{j}}\right]=-\left.\frac{\partial^{2} C(x)}{\partial x_{i} \partial x_{j}}\right|_{x=0}
$$




\subsection{Expectations}

To tackle the integral geometry of the random field, $Y$, suitable regularity conditions should be valid on $Y$. The subset $S$, in this paper, is restricted to being a bounded $d$-dimensional rectangle of $\mathbb{R}^{d},(d=2)$, of the form:

$$
S=\prod_{i=1}^{d}\left[0, R_{i}\right], \quad R_{i} \in \mathbb{R}^{+} \backslash\{0\}
$$

The random field, $Y$, defined on $S$, is said to be suitably regular if the following three conditions reported in (Adler, 1981; Alder and Taylor, 2003), for $S \subset \mathbb{R}^{2}$, are satisfied:

1. $Y$ has continuous partial derivatives of up to second order in an open neighbourhood of $S$.

2. The critical points ${ }^{1}$ of $Y_{\mid \partial_{k} S}$ are non-degenerate ${ }^{2}$ for $k=0,1,2$.

3. $Y_{\mid \partial_{k} S}$ has no critical points on $\bigcup_{i=0}^{k-1} \partial_{i} S$ for all $k=1,2$.

These conditions mean that the boundary of the excursion set $E_{h}$ at a given level $h$, $\left(\partial E_{h}=\{x \in S: Y(x)=h\}\right)$, and its intersections with the boundary of $S,(\partial S)$, are both suitably smooth.

For the Gaussian random field case, these conditions are satisfied, (see (Adler, 1981)), and so for the student's $t$ random fields with $\nu>2$, (see Worsley (1994), lemma 4.2). Thus, the linear combination of these random fields is considered suitably regular on $S$ for $\nu>2$.

\footnotetext{
${ }^{1}$ The points $x \in S$ for which $\nabla\left(Y_{\mid \partial_{k} S}\right)=0$.

${ }^{2} \operatorname{det} \nabla^{2}\left(Y_{\mid \partial_{k} S}\right) \neq 0$
} 
The expected Euler-Poincaré characteristic, denoted by $\mathbb{E}\left[\chi\left(E_{h}(Y, S)\right)\right]$, of the excursion set, $E_{h}(Y, S)$, of the $G T_{\beta}^{\nu}$ random field, $Y$, on $\mathbb{R}^{2}$, at a given threshold $h$, is expressed, (Adler, 1981; Hasofer, 1978), as:

$$
\mathbb{E}\left[\chi\left(E_{h}(Y, S)\right)\right]=\sum_{j=0}^{2} \mathcal{L}_{j}(S) \rho_{j}(h)
$$

where $\mathcal{L}_{j}(S)$ is the $j$-th dimensional Minkowski functional of $S$, such that $\mathcal{L}_{0}=1$ is the Euler-Poincare characteristic of $S, \mathcal{L}_{1}=R_{1}+R_{2}$ is half the boundary length of $S$, and $\mathcal{L}_{2}=R_{1} \times R_{2}$ is the two dimensional area of $S$. The coefficients $\rho_{j}(h)$, $(j=0,1,2)$, are called the $j$-th dimensional Euler-Poincaré, or Euler, characteristic intensities, (Alder and Taylor, 2003; Worsley, 1997), of the excursion set.

Theorem 1. The $j$-th dimensional Euler characteristic intensities, $\rho_{j}(),. j=0,1,2$ for an isotropic $G T_{\beta}^{\nu}$ random field on $\mathbb{R}^{2}$, with $\nu$ degrees of freedom, $\nu>2$, and $\beta>0$, for a given $h$, are expressed as follows:

(i) $\rho_{0}(h)=\mathbb{P}[Y \geq h]=\mathbb{E}\left[\mathbb{P}\left[\beta T^{\nu} \geq h-G \mid G\right]\right]$

$$
=\int_{h}^{\infty} \int_{-\infty}^{\infty}\left(1+\frac{(h-u)^{2}}{\beta^{2} \nu}\right)^{-\frac{\nu+1}{2}} e^{-u^{2} / 2} d u
$$

(ii) $\rho_{1}(h)=\frac{\lambda^{1 / 2}}{(2 \pi)^{3 / 2}} \int_{-\infty}^{\infty}\left(1+\frac{(h-u)^{2}}{\beta^{2} \nu}\right)^{-\frac{\nu-1}{2}} e^{-u^{2} / 2} d u$

$$
+\frac{\lambda_{G}^{1 / 2} \Gamma\left(\frac{\nu+1}{2}\right)}{(2 \pi)^{3 / 2} \beta \sqrt{\nu} \Gamma\left(\frac{\nu}{2}\right)} \int_{-\infty}^{\infty}\left(1+\frac{(h-u)^{2}}{\beta^{2} \nu}\right)^{-\frac{\nu+1}{2}} e^{-u^{2} / 2} d u
$$

$$
\text { (iii) } \begin{aligned}
\rho_{2}(h) & =\beta \frac{2^{\frac{1}{2}} \lambda \Gamma\left(\frac{\nu+1}{2}\right)}{(2 \pi)^{2} \Gamma\left(\frac{\nu}{2}\right)} \int_{-\infty}^{\infty} \frac{(h-u)}{\beta \sqrt{\nu}}\left(1+\frac{(h-u)^{2}}{\beta^{2} \nu}\right)^{-\frac{\nu-1}{2}} e^{-u^{2} / 2} d u \\
& +\frac{\lambda_{G} \Gamma\left(\frac{\nu+1}{2}\right)}{(2 \pi)^{2} \Gamma\left(\frac{\nu}{2}\right) \beta \sqrt{\nu / 2}} \int_{-\infty}^{\infty} u\left(1+\frac{(h-u)^{2}}{\beta^{2} \nu}\right)^{-\frac{\nu+1}{2}} e^{-u^{2} / 2} d u
\end{aligned}
$$

where $\Lambda_{G}=\lambda_{G} I_{2}$, and $\Lambda=\lambda I_{2}$. 
Proof. The proof of the theorem is based on previous theorems and lemmas given in (Adler, 1981; Adler and Taylor, 2007; Cao and Worsley, 1999; Worsley, 1995, 1994), on $\mathbb{R}^{d}$, (for not losing the generality).

For a suitably regular $G T_{\beta}^{\nu}$ random field, $Y$, and under the assumption that the conditional density of $Y, \dot{Y}_{1}, \ldots, \dot{Y}_{d-1}$ given $\dot{Y}_{d}$ is bounded above, and all the second order partial derivatives of $Y,\left\{\ddot{Y}_{k l}, 1 \leq k \leq d, 1 \leq l \leq d\right\}$ and $\dot{Y}_{d}$ have finite variance conditional on $Y, \dot{Y}_{1}, \ldots, \dot{Y}_{d-1}$, (Adler, 1981; Worsley, 1995), the $j$-th dimensional Euler characteristic intensity, $\rho_{j}(h)$, of the excursion set, $E_{h}(Y, S)$ can be expressed as follows :

$$
\rho_{j}(h)=(-1)^{j-1} \mathbb{E}\left[\dot{Y}_{j}^{+} \operatorname{det}\left(\ddot{Y}_{\mid j-1}\right) \mid \dot{Y}_{\mid j-1}=0, Y=h\right] p_{\dot{Y}_{\mid j-1}}(0, h)
$$

where the term $\mid j-1$ represents the sub-matrix of the first $j-1$ rows and columns, and $j$ refers to the matrix $j$-th component. $p_{\dot{Y}_{\mid j-1}}(0, h)$ is the joint probability density of $\dot{Y}_{\mid j-1}$ at zero and $Y=h$, and $\dot{Y}^{+}=\dot{Y}$ when $\dot{Y}>0$.

We will use in our proof, the conditional expectations and we will express the Euler characteristic intensities of $Y$ conditioning on the Gaussian random field $G$.

Conditioning on $G$, the random field $Y$ becomes a student's $t$ random field with $\nu$ degrees of freedom, and $\rho_{j}(h)$ in equation (16) can be expressed as:

$$
\rho_{j}(h)=\mathbb{E}\left\{\rho_{j}^{T^{\nu}}(h) \mid G, Y=h\right\}
$$

where $\rho_{j}^{T^{\nu}}(h)$ is the $j$-th dimensional Euler characteristic intensity of the student's $t$ random field, $T^{\nu}$, expressed by Worsley (1994). In order to give the explicit formula of the $\rho_{j}(h)$, the first and second order partial derivatives of $Y$ should be expressed. The following results due to Worsley (1994) and Adler (1981) are used for this aim: 
Lemma 1 (Adler (1981)). Let $G$ be a Gaussian random field, (at any fixed point $x \in S)$, then, $\dot{G} \sim \operatorname{Normal}_{d}\left(0, \Lambda_{G}\right)$ independent of $G$ and $\ddot{G}$

Conditioning on $G$,

$$
\ddot{G} \mid G \sim \operatorname{Normal}_{d \times d}\left(-G \Lambda_{G}, M\left(\Lambda_{G}\right)\right)
$$

where $M\left(\Lambda_{G}\right)=\operatorname{Cov}\left(\nabla^{2} G \mid G\right)$ is $(d \times d)$ symmetric.

Lemma 2 (Worsley (1994)). The first and second order partial derivatives of the student's $t$ random field with $\nu$ degrees of freedom, $T^{\nu}$, (at any fixed point $x$ ), can be expressed in term of independent random random variables as follows:

$$
\begin{aligned}
& \dot{T}^{\nu}=\nu^{1 / 2}\left(1+\left(T^{\nu}\right)^{2} / \nu\right) W^{-1 / 2} z_{1} \\
& \ddot{T}^{\nu}=\nu^{1 / 2}\left(1+\left(T^{\nu}\right)^{2} / \nu\right) W^{-1}\left\{-\nu^{-1 / 2} T^{\nu}\left(Q-2 z_{1} z_{1}^{t}\right)-z_{1} z_{2}^{t}-z_{2} z_{1}^{t}+W^{1 / 2} H\right\}
\end{aligned}
$$

where $W \sim \chi_{\nu+1}^{2}, z_{1}, z_{2} \sim \operatorname{Normal}_{d}(0, \Lambda), Q \sim \operatorname{Wishart}_{d}(\Lambda, \nu-1)$ and $H \sim$ Normal $_{d \times d}(0, M(\Lambda))$, all are independent.

Consequently, the first and second order partial derivatives of $Y$ can be also expressed in term of independent random variables, at any fixed point $x \in S$, as follows:

$$
\begin{aligned}
& \dot{Y}=\dot{G}+\beta \nu^{1 / 2}\left(1+\frac{(Y-G)^{2}}{\beta^{2} \nu}\right) W^{-1 / 2} z_{1} \\
& \ddot{Y}=-G \Lambda_{G}+V \\
& +\beta \nu^{1 / 2}\left(1+(Y-G)^{2} / \nu\right) W^{-1}\left\{-\beta^{-1} \nu^{-1 / 2}(Y-G)\left(Q-2 z_{1} z_{1}^{t}\right)-z_{1} z_{2}^{t}-z_{2} z_{1}^{t}+W^{1 / 2} H\right\}
\end{aligned}
$$

where $V \sim \operatorname{Normal}_{d \times d}\left(0, M\left(\Lambda_{G}\right)\right)$, and $z_{1}, z_{2}, W, Q, H, G$ are all independent.

Conditioning on $G, Y$ and $W$, the $j$-th dimensional Euler characteristic density 
$\rho_{j}^{T^{\nu}}(h)$, in our case, becomes:

$$
\rho_{j}^{T^{\nu}}(h)=\mathbb{E}_{W}\left\{\mathbb{E}\left[\left(\dot{Y}_{j}^{+}\right) \operatorname{det}\left(-\ddot{Y}_{\mid j-1}\right) \mid \dot{Y}_{\mid j-1}=0, G, W, Y=h\right] p_{\dot{Y}_{\mid j-1}}(0, W, G, h)\right\}
$$

where $p_{\dot{Y}_{\mid j-1}}(0, W, G, h)$ is the joint probability density of $\dot{Y}_{\mid j-1}$ at zero conditional on $Y=h, W$ and $G$.

The matrix $\ddot{Y}_{\mid j-1}$ is the $(j-1) \times(j-1)$ of the Hessian matrix $\nabla^{2} Y$ with respect to $x_{1}, \ldots, x_{j-1}$. Considering the conditioning on $\dot{Y}_{1}=0, \ldots, \dot{Y}_{j-1}=0$, then, the $(j-1)$ first order partial derivative components of both $\dot{G}$ and $z_{1}$ are zeros, i.e., $\left(\dot{G}_{1}=0, \ldots, \dot{G}_{j-1}=0\right)$ and $\left(z_{1_{(1)}}=0, \ldots, z_{1_{(j-1)}}=0\right)$, since $\dot{G}$ and $z_{1}$ are independent. Hence, $\ddot{Y}_{\mid j-1}$ can be written as:

$$
\ddot{Y}_{\mid j-1}=-G \Lambda_{G}+V+\beta \nu^{1 / 2}\left(1+(Y-G)^{2} / \nu\right) W^{-1}\left\{-\beta^{-1} \nu^{-1 / 2}(Y-G) Q+W^{1 / 2} H\right\}
$$

Furthermore, $\ddot{Y}_{\mid j-1}$ and $\dot{Y}_{j}$ are also independent conditioning on $G, Y, W$ and $\dot{Y}_{1}=$ $0, \ldots, \dot{Y}_{j-1}=0$, so:

$$
\begin{aligned}
& \mathbb{E}\left[\left(\dot{Y}_{j}^{+}\right) \operatorname{det}\left(-\ddot{Y}_{\mid j-1}\right) \mid \ddot{Y}_{\mid j-1}=0, Y=h\right]=(-1)^{j-1} \times \\
& \mathbb{E}\left[\left(\dot{Y}_{j}^{+}\right) \mid \dot{Y}_{\mid j-1}=0, G, W, Y=h\right] \times \mathbb{E}\left[\operatorname{det}\left(\ddot{Y}_{\mid j-1}\right) \mid \dot{Y}_{\mid j-1}=0, G, W, Y=h\right]
\end{aligned}
$$

Since $\dot{Y}$, conditioning on $G, Y$ and $W$, is a linear mixture of two independent Gaussian random fields, $\dot{G}$ and $z_{1}$, with covariance matrix $\left(\Lambda_{G}+\beta^{2} \nu\left(1+\frac{(h-G)^{2}}{\beta^{2} \nu}\right)^{2} W^{-1} \Lambda\right)$, then:

$\mathbb{E}\left[\left(\dot{Y}_{j}^{+}\right) \mid \dot{Y}_{\mid j-1}=0, G, W, Y=h\right]=(2 \pi)^{-1 / 2}\left\{\lambda_{G_{(j)}}+\beta^{2} \nu\left(1+\frac{(h-G)^{2}}{\beta^{2} \nu}\right)^{2} W^{-1} \lambda_{j}\right\}^{1 / 2}$

where $\lambda_{G_{(j)}}, \lambda_{j}$ are the $j$-th elements of the diagonal matrix $\Lambda_{G}, \Lambda$, respectively, and due to isotropy they become $\lambda_{G_{(j)}}=\lambda_{G}$ and $\lambda=\lambda_{j}$. 
In order to calculate the determinant $\operatorname{det}\left(\ddot{Y}_{\mid j-1}\right)$ conditional on $Y=h, G, W$ and $\dot{Y}_{\mid j-1}=0$, the matrix $\ddot{Y}_{\mid j-1}$ is expressed as:

$$
\ddot{Y}_{\mid j-1}=B+a Q+b H
$$

where $a=\beta \nu^{1 / 2}\left(1+(Y-G)^{2} / \nu\right) W^{-1}\left\{-\beta^{-1} \nu^{-1 / 2}(Y-G), b=W^{1 / 2}\right.$ are constants, $B \sim \operatorname{Normal}_{(j-1) \times(j-1)}\left(-G \Lambda_{G}, M\left(\Lambda_{G}\right)\right), Q \sim \operatorname{Wishart}(\Lambda, \nu-1)$, and $H \sim$ $\operatorname{Normal}_{(j-1) \times(j-1)}(0, M(\Lambda))$.

Firstly, let consider the orthogonal $(j-1) \times(j-1)$ matrix, $U$, such that $U^{t} \Lambda_{\mid j-1} U=$ $I_{j-1}$, then:

$$
\mathbb{E}\left[\operatorname{det}\left(\ddot{Y}_{\mid j-1}\right) \mid \dot{Y}_{j-1}=0, Y=h, G, W\right]=\mathbb{E}[\operatorname{det}(\tilde{B}+a \tilde{Q}+b \tilde{H})]
$$

where $\tilde{B}=U^{t} B U$, and $\tilde{B} \sim \operatorname{Normal}_{(j-1) \times(j-1)}\left(-U^{t} G \Lambda_{G} \Lambda^{-1} U^{t}, M\left(U^{t} \Lambda_{G} \Lambda^{-1} U^{t}\right)\right)$, $\tilde{Q} \sim \operatorname{Wishart}\left(I_{\mid j-1}, \nu-1\right)$, and $\tilde{H} \sim \operatorname{Normal}_{(j-1) \times(j-1)}(0, M(I))$.

Using the lemmas in the appendix, (Appendix A), we obtain:

$$
\begin{aligned}
\mathbb{E}\left[\operatorname{det}\left(\ddot{Y}_{\mid j-1}\right) \mid \dot{Y}_{j-1}=0, Y=h, G, W\right] & =\operatorname{det}_{j-1}(\Lambda) \sum_{i=0}^{\lfloor(j-1) / 2\rfloor} \frac{(-1)^{i}}{2^{i} i !} b^{2 i} \sum_{k=0}^{j-1-2 i} a^{k}\left(\begin{array}{c}
\nu-1 \\
k
\end{array}\right) \\
& \times[(2 i+k) !] \operatorname{detr}_{j-1-2 i-k}(\tilde{B})
\end{aligned}
$$

where $\operatorname{det}_{k}($.$) stands for the matrix determinant of the first (k \times k)$ elements, $\operatorname{detr}_{k}$ is the sum of the determinants of all $k \times k$ principal minors, and

$$
\operatorname{detr}_{m}(\tilde{B})=\operatorname{det}_{m}\left(\Lambda_{G}\right) \operatorname{det}_{m}\left(\Lambda^{-1}\right) \sum_{i=0}^{\lfloor(m) / 2\rfloor} \frac{(-1)^{m-i}(2 i !)}{2^{i} i !} G^{m-2 i}
$$


Putting equations (25) and (24) together yields:

$$
\begin{aligned}
& \mathbb{E}\left[\operatorname{det}\left(\ddot{Y}_{\mid j-1}\right) \mid \dot{Y}_{j-1}=0, Y=h, G, W\right]=\operatorname{det}_{j-1}(\Lambda) \sum_{i=0}^{\lfloor(j-1) / 2\rfloor} \frac{(-1)^{i}}{2^{i} i !} b^{2 i} \sum_{k=0}^{j-1-2 i} a^{k}\left(\begin{array}{c}
\nu-1 \\
k
\end{array}\right) \\
& \times \operatorname{det}_{j-1-2 i-k}\left(\Lambda_{G} \Lambda^{-1}\right) \sum_{l=0}^{\lfloor(j-1-2 i-k) / 2\rfloor} \frac{(-1)^{j-1-2 i-k-l}}{2^{l} l !}(2 i+2 l+k) ! G^{j-2 i-k-2 l-1}
\end{aligned}
$$

The joint probability density function of the $(j-1)$ first derivatives of $\dot{Y}, p_{\dot{Y}_{\mid j-1}}(\dot{Y}, G, W, h)$, in equation (18), conditioning on $G, W, Y=h$, is a Gaussian joint probability density, $\left(\dot{Y}_{\mid j-1} \sim \operatorname{Normal}_{j-1}\left(0, \Lambda_{G}+\beta^{2} \nu\left(1+(h-G)^{2} / \beta^{2} \nu\right)^{2} W^{-1} \Lambda\right)\right)$. Hence, the density of $\dot{Y}_{\mid j-1}$ at zero becomes:

$$
p_{\dot{Y}_{\mid j-1}}(0, G, W, h)=(2 \pi)^{-\frac{j-1}{2}}\left\{\operatorname{det}_{j-1}\left(\Lambda_{G}+\beta^{2} \nu\left(1+(h-G)^{2} / \beta^{2} \nu\right)^{2} W^{-1} \Lambda\right)\right\}^{-\frac{1}{2}}
$$

Recall that $W \sim \chi_{\nu+1}^{2}$ is a Chi-squared random field with $\nu+1$ degrees of freedom, So, its moments are given such that:

$$
\mathbb{E}\left[W^{j}\right]=2^{j} \frac{\Gamma((\nu+1) / 2+j)}{\Gamma((\nu+1) / 2)}
$$

Since the $j$-th dimensional Euler characteristic intensity of the student's $t$ excursion set conditioning on the Gaussian random field, $\rho_{j}^{T^{\nu}}(h)$, is known now, the $j$-th dimensional Euler characteristic intensity of the $G T_{\beta}^{\nu}$ excursions set, $\rho_{j}(h)$, can then be expressed using equations (17) and (18), such that:

$$
\begin{aligned}
& \rho_{j}(h)=(-1)^{j} \int_{-\infty}^{\infty} p_{G}(u) d u \times \\
& \mathbb{E}_{W}\left\{\mathbb{E}\left[\left(\dot{Y}_{j}^{+}\right) \operatorname{det}\left(\ddot{Y}_{\mid j-1}\right) \mid \dot{Y}_{\mid j-1}=0, G, W, Y=h\right] p_{\dot{Y}_{\mid j-1}}(0, W, G, h)\right\} p_{Y}(h ; G)
\end{aligned}
$$

where $p_{G}(u)$ is the Gaussian probability density function, and $P_{Y}(h ; G)$ is the probability density function of $Y$ at $h$ conditional on $G$, which becomes a student's $t$ 
density with $\nu$ degrees of freedom:

$$
p_{Y}(h ; G)=\frac{\Gamma\left(\frac{\nu+1}{2}\right)}{\beta \sqrt{\nu} \Gamma\left(\frac{\nu}{2}\right)}\left(1+\frac{(h-G)^{2}}{\beta^{2} \nu}\right)^{-\frac{\nu+1}{2}}
$$

For $j=2$,

$$
\begin{aligned}
& \rho_{2}(h)=\beta \frac{2^{\frac{1}{2}} \lambda_{X} \Gamma\left(\frac{\nu+1}{2}\right)}{(2 \pi)^{2} \Gamma\left(\frac{\nu}{2}\right)} \int_{-\infty}^{\infty} \frac{(h-u)}{\beta \sqrt{\nu}}\left(1+\frac{(h-u)^{2}}{\beta^{2} \nu}\right)^{-\frac{\nu-1}{2}} e^{-u^{2} / 2} d u \\
& +\frac{\lambda_{G} \Gamma\left(\frac{\nu+1}{2}\right)}{(2 \pi)^{2} \Gamma\left(\frac{\nu}{2}\right) \beta \sqrt{\nu / 2}} \int_{-\infty}^{\infty} u\left(1+\frac{(h-u)^{2}}{\beta^{2} \nu}\right)^{-\frac{\nu+1}{2}} e^{-u^{2} / 2} d u
\end{aligned}
$$

For $j=1$, The expectation $\mathbb{E}\left[\dot{Y}_{j}^{+} \mid \dot{Y}_{\mid j-1}=0, Y=h, G, W\right]$ is expressed by using the condition that stats $\dot{Y}>0$ when $\dot{G}>0$ and $z_{1}>0$, such that:

$$
\begin{aligned}
\mathbb{E}\left[\dot{Y}_{j}^{+} \mid \dot{Y}_{\mid j-1}=0, Y=h, G, W\right] & =\mathbb{E}\left[\dot{G}_{j}^{+} \mid \dot{Y}_{\mid j-1}=0, Y=h, G, W\right]+ \\
& \beta \nu^{1 / 2}\left(1+\frac{(h-G)^{2}}{\beta^{2} \nu}\right) W^{-1 / 2} \mathbb{E}\left[z_{1_{j}}^{+} \mid \dot{Y}_{\mid j-1}=0, Y=h, G, W\right] \\
& =(2 \pi)^{-1 / 2}\left[\lambda_{G}^{1 / 2}+\beta \nu^{1 / 2}\left(1+\frac{(h-G)^{2}}{\beta^{2} \nu}\right) W^{-1 / 2} \lambda^{1 / 2}\right]
\end{aligned}
$$

Then

$$
\begin{aligned}
\rho_{1}(h) & =(2 \pi)^{-1 / 2} \int_{-\infty}^{\infty} \mathbb{E}_{W}\left[\lambda_{G}^{1 / 2}+\beta \nu^{1 / 2}\left(1+\frac{(h-G)^{2}}{\beta^{2} \nu}\right) W^{-1} \lambda^{1 / 2}\right] p_{Y}(h ; G) p_{G}(u) d u \\
& =\frac{\lambda^{1 / 2}}{(2 \pi)^{3 / 2}} \int_{-\infty}^{\infty}\left(1+\frac{(h-u)^{2}}{\beta^{2} \nu}\right)^{-\frac{\nu-1}{2}} e^{-u^{2} / 2} d u \\
& +\frac{\lambda_{G}^{1 / 2} \Gamma\left(\frac{\nu+1}{2}\right)}{(2 \pi)^{3 / 2} \beta \nu^{1 / 2} \Gamma\left(\frac{\nu}{2}\right)} \int_{-\infty}^{\infty}\left(1+\frac{(h-u)^{2}}{\beta^{2} \nu}\right)^{-\frac{\nu+1}{2}} e^{-u^{2} / 2} d u
\end{aligned}
$$

Finally, for $j=0, \rho_{0}(h)=\mathbb{P}[Y \geqslant h]$. 
Corollary 1. The expected Euler-Poincaré characteristic of the excursion set, $E_{h}(Y, S)$, above a level $h$ where it does not touch the boundaries of $S=\left[0, R_{1}\right] \times\left[0, R_{2}\right]$ is:

$$
\begin{aligned}
\mathbb{E}\left[\chi\left(E_{h}(Y, S)\right)\right] & =R_{1} R_{2} \beta \frac{2^{\frac{1}{2}} \lambda \Gamma\left(\frac{\nu+1}{2}\right)}{(2 \pi)^{2} \Gamma\left(\frac{\nu}{2}\right)} \int_{-\infty}^{\infty} \frac{(h-u)}{\beta \sqrt{\nu}}\left(1+\frac{(h-u)^{2}}{\beta^{2} \nu}\right)^{-\frac{\nu-1}{2}} e^{-u^{2} / 2} d u \\
& +R_{1} R_{2} \frac{\lambda_{G} \Gamma\left(\frac{\nu+1}{2}\right)}{(2 \pi)^{2} \Gamma\left(\frac{\nu}{2}\right) \beta \sqrt{\nu / 2}} \int_{-\infty}^{\infty} u\left(1+\frac{(h-u)^{2}}{\beta^{2} \nu}\right)^{-\frac{\nu+1}{2}} e^{-u^{2} / 2} d u
\end{aligned}
$$

From the last result, one can notice that the expected Euler-Poincaré characteristic of the $G T_{\beta}^{\nu}$ excursion set, at high threshold $(h \longrightarrow \infty)$, becomes dependent on the covariance structure of the student's $t$ random field related to $\lambda$ such that:

$\mathbb{E}\left[\chi\left(E_{h}(Y, S)\right)\right] \cong R_{1} R_{2} \beta \frac{2^{\frac{1}{2}} \lambda \Gamma\left(\frac{\nu+1}{2}\right)}{(2 \pi)^{2} \Gamma\left(\frac{\nu}{2}\right)} \int_{-\infty}^{\infty} \frac{(h-u)}{\beta \sqrt{\nu}}\left(1+\frac{(h-u)^{2}}{\beta^{2} \nu}\right)^{-\frac{\nu-1}{2}} e^{-u^{2} / 2} d u+O\left(\frac{1}{h}\right)$

when $h \longrightarrow \infty$.

\section{Application}

A 3D rough anisotropic surface has been measured by a non-contact white light interferometry as seen in figure 1(a). The 3D height's map is observed over a compact subset $S$ of $0.86 \times 0.86 \mathrm{~mm}^{2}$ size, with spatial resolution $1.8 \mu \mathrm{m}$ in both $x$ and $y$ directions. The surface is considered realized from the combination of anisotropic Gaussian random field and a homogeneous student's $t$ one. The Gaussian component is obtained from the convolution between the white Gaussian noise with a cosine kernel with wavelengths $\left(\lambda_{G_{1}}\right.$, and $\left.\lambda_{G_{2}}\right)$, which are estimated using the spectral representation such that $\lambda_{G_{1}}=15 \mathrm{~mm}^{-2}, \lambda_{G_{2}}=117 \mathrm{~mm}^{-2}$. Furthermore, the surface is averaged and divided to its standard deviation in order to get a zero mean and unit 
variance $G T_{\beta}^{\nu}$ random field. The excursion sets above high thresholds, $(h>2.5)$, are considered isotropic, so the parameters $\beta, \nu$, and $\lambda$ are estimated numerically using the expected Euler-Poincaré characteristic in equation (35), and obtained $\nu=5$, $\beta=0.2$ and $\lambda=190.3 \mathrm{~mm}^{-2}$. The expected and the empirical Euler-Poincaré characteristics of the $G T_{\beta}^{\nu}$ excursion set and the surface upcrossings above $h$, respectively, are compared, (see figure 1(b)). The surface roughness is related to the peaks, and

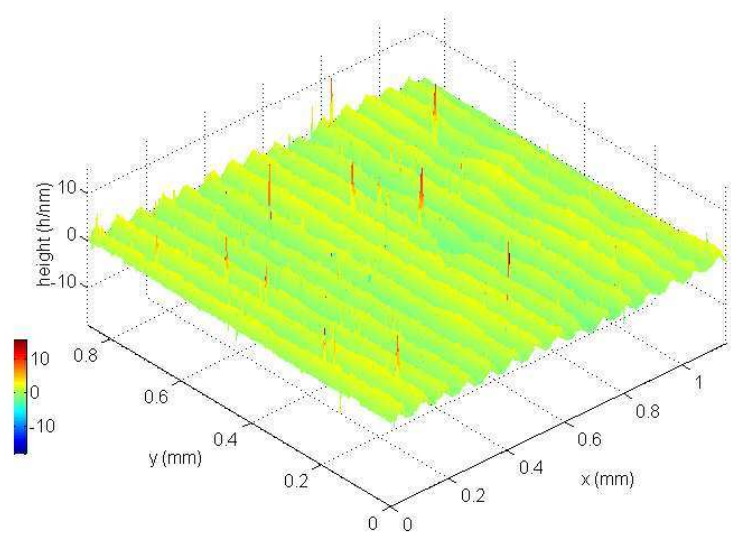

(a)

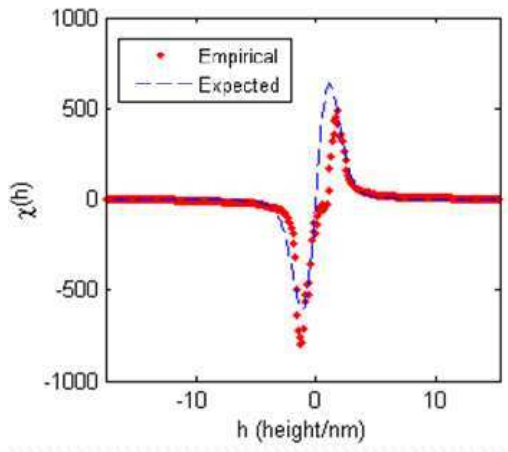

(b)

Figure 1: (a) A real 3D rough surface observed from a plastic material (UHMWPE) involved in the hip replacement. (b) Fitting the empirical, (red curve), and the expected, (blue curve), Euler-Poincaré characteristic between the real surface upcrossings, and the $G T_{0.2}^{5}$ excursion sets, respectively.

it corresponds to the number of the global maximums and minimums $\mathbb{P}\left[Y_{\max }>h\right]$, $\mathbb{P}\left[Y_{\min }<h\right]$, respectively, which are approximated by the expected Euler-Poincaré characteristic at high thresholds (Adler, 1981; Hasofer, 1978):

$$
\begin{aligned}
& \mathbb{P}\left[Y_{\text {max }}>h\right] \approx \mathbb{E}\left[\chi\left(E_{h}(Y, S)\right)\right] \\
& \mathbb{P}\left[Y_{\text {min }}<h\right] \approx \mathbb{E}\left[\chi\left(E_{-h}(-Y, S)\right)\right]=-\mathbb{E}\left[\chi\left(E_{h}(Y, S)\right)\right]
\end{aligned}
$$


Since the expected Euler characteristic above high thresholds, or below low thresholds, counts the number of connected components (isolated peaks or valleys). The comparison in this example shows that the expected number of the peaks and valleys can be estimated accurately from the expected Euler characteristic of the $G T_{\beta}^{\nu}$ random field which becomes close to the expected Euler-Poincaré characteristic of the student's $t$ random field at the high thresholds.

\section{Conclusion}

A random field, denoted by $G T_{\beta}^{\nu}$, and defined from the linear combination of the Gaussian random field and student's $t$ one with $\nu$ degrees of freedom scaled by $\beta$ is introduced in this paper. The expected Euler-Poincaré characteristic and Minkowski functionals of the $G T_{\beta}^{\nu}$ excursion sets are expressed analytically on a rectangle $S$ of $\mathbb{R}^{2}$.

An application is reported on a 3D rough surface of a finished UHMWPE plastic material observed by a non-contact interferometry. Fitting the empirical and the expected Euler-Poincaré charcaterisitc enabled describing the surface roughness. The future work will focus on studying more flexible random fields including further significant parameters for modeling the rough surfaces such as the family of the skewed random fields.

\section{Appendix A. Lemmas for the proof of the Theorem 1}

Lemma 3 (Worsley (1994)). Let $H \sim \operatorname{Normal}_{d \times d}(0, M)$ and let $B$ be a fixed symmetric $d \times d$ matrix. Then

$$
\mathbb{E}[\operatorname{det}(B+H)]=\sum_{j=0}^{\lfloor d / 2\rfloor} \frac{(-1)^{j}(2 j) !}{2^{j}} \operatorname{detr}_{d-2 j}(B)
$$


Lemma 4 (Cao and Worsley (1999)). Let $Q \sim W \operatorname{Wishart}\left(I_{d}, \nu\right), H \sim \operatorname{Normal}_{d \times d}(0, M)$ are independent, and $B$ be a fixed symmetric $d \times d$ matrix. Let $a, b$ be fixed scalars. Then

$$
\mathbb{E}[\operatorname{det}(B+a Q+b H)]=\sum_{j=0}^{\lfloor d / 2\rfloor} \frac{(-1)^{j}}{2^{j} j !} b^{2 j} \sum_{k=0}^{d-2 j} a^{k}\left(\begin{array}{l}
\nu \\
k
\end{array}\right)(2 j+k) ! \operatorname{detr}_{d-2 j-k}(B)
$$

Adler, R., Taylor, J., july 2011. Topological complexity of smooth random functions: École d'Été de Probabilités de Saint-Flour XXXIX-2009. Springer Berlin Heidelberg.

Adler, R. J., June 1981. The Geometry of Random Fields. John Wiley \& Sons Inc.

Adler, R. J., Taylor, J., Jun. 2007. Random Fields and Geometry, 1st Edition. Springer.

Adler, R. J., Taylor, J. E., Worsley, K. J., 2010. Applications of random fields and geometry: Foundations and case studies.

Alder, R. J., Taylor, J. E., 2003. Random fields and their geometry. Birkhäuser, Boston.

Cao, J., 1997. Excursion sets of random fields with applications to human brain mapping. Ph.D. thesis, Departement of Mathematics and Statistics, McGill University, Quebec, Canada.

Cao, J., Worsley, K., 2001. Applications of random fields in human brain mapping. Springer lecture notes in statistics 159, 169-182.

Cao, J., Worsley, K. J., 1999. The detection of local shape changes via the geometry of Hotelling's $t^{2}$ fields. The Annals of Statistics 27 (3), 925-942. 
Hasofer, A. M., Mar. 1978. Upcrossings of random fields. Advances in Applied Probability 10, 14 .

Worsley, K., 1995. Boundary corrections for the expected euler characteristic of excursion sets of random fields, with an application to astrophysics. Advances in Applied Probability 27, 943-959.

Worsley, K., 1997. The geometry of random fields. Chance 9 (1), 27-40.

Worsley, K. J., 1994. Local maxima and the expected euler characteristic of excursion sets of $\chi^{2}, \mathrm{~F}$ and $t$ fields. Advances in Applied Probability 26 (1), 13-42. 